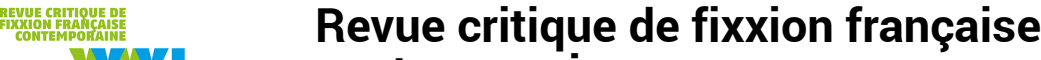 \\ XXI contemporaine

Radicalités

\section{Pour une poétique destituante}

Lefebvre, Lordon, Quintane

Julien Jeusette

\section{(2) OpenEdition}

Journals

Édition électronique

URL : https://journals.openedition.org/fixxion/579

DOI : $10.4000 /$ fixxion. 579

ISSN : 2295-9106

Éditeur

Ghent University

Référence électronique

Julien Jeusette, "Pour une poétique destituante », Revue critique de fixxion française contemporaine [En ligne], 20 | 2020, mis en ligne le 15 juin 2020, consulté le 17 mai 2022. URL : http://

journals.openedition.org/fixxion/579; DOI : https://doi.org/10.4000/fixxion.579

Ce document a été généré automatiquement le 17 mai 2022.

\section{cc)}

Les contenus de la Revue critique de fixxion française contemporaine sont mis à disposition selon les termes de la licence Creative Commons Attribution - Pas d'Utilisation Commerciale - Pas de Modification 4.0 International. 


\title{
Pour une poétique destituante
}

\author{
Lefebvre, Lordon, Quintane
}

Julien Jeusette

\begin{abstract}
non pas "surveiller son langage" (au contraire!)
mais maintenir son état de veille dans le langage, et tenir par-là même que le langage soit une

veille

(Jean-Christophe Bailly, L'élargissement du poème)
\end{abstract}

1 Dès la fin de la Seconde Guerre mondiale, Victor Klemperer, un philologue allemand, se met à trier ses carnets de notes afin d'en faire un livre. Déchu de son poste à l'université de Dresde peu après l'accession au pouvoir d'Hitler, il est sauvé de la déportation grâce à son mariage avec une "non-juive". Envoyé à l'usine, privé de livres, il ne peut mener à bien ses travaux (un ouvrage sur la littérature française du XVIII ${ }^{e}$ siècle), mais résiste à l'abrutissement en se levant au milieu de la nuit pour écrire. Pendant douze ans, entre 1933 et 1945, il analyse minutieusement, d'un point de vue philologique, la manière dont les Nazis transforment la langue allemande. Les changements constatés sont tellement profonds que Klemperer baptise le monstre : LTI, Lingua Tertii Imperii. Cette langue appauvrie, qui se propage par répétition quotidienne dans les discours, les journaux et les livres autorisés, a une particularité : elle s'impose de manière uniforme à tous les Allemands, qui en adoptent plus ou moins involontairement les tournures et le style.

2 Le philologue rend compte de cette diffusion généralisée dans une scène frappante, qui le blesse davantage que les agressions verbales de la Gestapo : Elsa, son ancienne assistante, brillante germaniste qui l'aide durant la guerre grâce à la position de son mari ${ }^{1}$ est irrémédiablement contaminée. Au cours d'une discussion sur la pédagogie, elle dit vouloir une éducation juive pour ses enfants, mais espère qu'ils deviendront également "des Allemands fanatiques" balbutie, avant de lui faire voir la gravité de ses mots: "Ne savez-vous pas que vous parlez la langue de votre ennemi mortel, et qu'ainsi vous vous avouez vaincue, et qu'ainsi vous vous livrez. [...] Si vous ne le savez pas, vous qui êtes une femme lettrée, [...] qui donc le sentira et l'évitera ?"3. Elsa admet sa faute, s'excuse et promet d'y être 
attentive, mais au rendez-vous suivant, elle emploie involontairement d'autres expressions de la LTI. Lorsque Klemperer réitère son indignation, elle ne le prend plus au sérieux et lui reproche son intransigeance. En d'autres termes, les persécutés du nazisme sont eux-mêmes imprégnés de leur langue au point de la juger naturelle ${ }^{4}$ comment dès lors résister?

3 La puissance linguistique par le biais de laquelle un groupe impose son monde n'est pas propre à l'idéologie hitlérienne, comme en fait l'expérience une poétesse française près d'un demi-siècle plus tard. Un jour, tombée par hasard sur une phrase issue des Mémoires du général de Gaulle - "La France vient du fond des âges" - Nathalie Quintane la déclame avec malice devant quelques amis. Elle s'attend à susciter le rire. Prononcer une telle affirmation à voix haute suffirait à en dégonfler l'emphase, à en faire entendre le ridicule. Or ses amis, qui partageaient jusque-là le même humour, n'ont pas la réaction escomptée : au lieu de s'en moquer, ils prennent la phrase au sérieux et considèrent qu'elle énonce une vérité. Le souffle coupé, l'écrivaine y voit le symptôme d'une situation nouvelle, pour le moins inquiétante :

cette phrase était forcément révélatrice d'un système de pensée : il y avait mon système de pensée, cet humour, qui me permettait de m'amuser à cette phrase et à d'autres, et il y avait le nouveau système de pensée de mes vieux amis. [...] J'étais restée dans l'Ancien Monde, et eux avaient basculé dans le Nouveau. ${ }^{5}$

4 Au début des "années 10 " - qui s'ouvrent d'ailleurs avec l'inscription polémique des mêmes Mémoires au programme du baccalauréat littéraire français -, la communauté de pensée à laquelle l'écrivaine croyait appartenir s'est imperceptiblement fracturée. Un certain imaginaire de gauche n'est plus partagé. Comme Klemperer, Quintane s'aperçoit avec stupeur que, sans s'en rendre compte, ceux qui se disent et s'imaginent les ennemis d'une idéologie particulière en sont eux-mêmes à la fois les dépositaires et les vecteurs.

5 Les prémisses du basculement évoqué par Quintane ont été étudiées en détail par Éric Hazan en 2006. Suivant l'exemple de Klemperer, il publie un essai intitulé $L Q R$, afin de décrire la "lingua quintae respublicae". Pleinement en vigueur à partir des années 1990, cette langue qui se propage dans les médias par le biais des économistes, des publicitaires, des cadres d'entreprise et des experts en communication est "à la fois

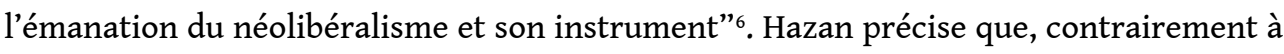
la LTI, la LQR n'est pas le fruit d'un bureau de propagande centralisé, mais émerge de différents secteurs qui ont tous intérêt à "escamoter le conflit", à anesthésier la population en faisant "régner le silence". Une fois diffusée assez largement, cette langue se reproduit elle-même et fonctionne alors comme "un écran sémantique permettant de faire tourner le moteur sans jamais en dévoiler les rouages".

Hazan s'emploie à décrire les tours et les ruses de la LQR, dont la force n'a pas faibli à ce jour ${ }^{10}$ : au niveau syntaxique, il relève notamment la prépondérance des tournures hyperboliques et des phrases-choc sans verbes ni articulations logiques; au niveau lexical, il met en évidence la manière dont certains termes sont répétés à satiété pour masquer le vide de leur référent (croissance, crise, réforme). La logique fondamentale de cette langue est l'euphémisme: "pauvre" est remplacé par "défavorisé", "exploité" par "exclu", "lutte entre patronat et syndicats" par "négociation entre partenaires sociaux", et ainsi de suite. Hazan montre à quel point les conséquences de ces altérations linguistiques sont délétères : comme la LTI, la LQR est une langue performative - elle "poétise et pense à ta place"11, se lamentait Klemperer en reprenant 
les fameux vers de Schiller. La langue du pouvoir néolibéral et sécuritaire, en imprégnant toutes les sphères de la société, crée ainsi l'illusion d'une cité unie, sans dissension ni conflit. Les mesures sociales les plus rétrogrades passent alors inaperçues et n'ont plus besoin d'être justifiées.

7 Toute langue impose certes une vision du monde particulière, mais en ayant un pied dans l'“Ancien Monde", ces trois penseurs sont capables d'entrevoir, du moins pour un temps, le remplacement d'un imaginaire par un autre. C'est la perception du changement qui les frappe et qui leur indique la nature toujours déjà politique de l'aliénation linguistique. La phrase lumineuse de Derrida, "Je n'ai qu'une langue, or ce n'est pas la mienne"12 prend ici un sens funeste : Nous n'avons qu'une langue, or c'est celle du pouvoir (totalitaire/néolibéral), constatent avec dégoût Klemperer, Quintane et Hazan $^{13}$.

8 La tâche la plus urgente est dès lors de nature philologique : pour l'historien allemand, il faut traquer les restes de LTI présents dans l'allemand d'après-guerre et les enterrer définitivement "dans la fosse commune" 14 ; pour les contemporains, il faut désactiver la LQR avant qu'elle ne devienne le monolinguisme du futur. Pour y parvenir, Klemperer et Hazan proposent le dévoilement : "Cohérente et mégaphonique, cette langue souffre pourtant d'un lourd handicap : elle ne doit surtout pas apparaître pour ce qu'elle est"15. En d'autres termes, il faudrait mettre en lumière les mensonges et les ruses de ces langues pour que s'effondre l'édifice social qu'elles soutiennent. Or l'exemple d'Elsa, l'ancienne assistante de Klemperer, montre bien que la connaissance n'est pas suffisante en elle-même. Comment faire "faire revenir (l'insu de) ce ridicule"16, se demande Quintane au sujet de la phrase du général de Gaulle? Quelle méthode adopter lorsque les individus sont captifs d'une langue fabriquée au point qu'elle constitue déjà leur subjectivité?

\section{Dramatiser la finance}

9 “On pourra analyser la crise financière sous toutes ses coutures, raffiner l'argument autant qu'on veut, démonter les systèmes, exposer les rouages, tout ça ne vaudra jamais une image bien choisie qui fait bouillir les sangs" ${ }^{17}$. À l'idée marxiste selon laquelle le savoir est au principe de la contestation ${ }^{18}$, Frédéric Lordon oppose l'efFicacité propre à l'esthétique : davantage que des arguments avancés de manière didactique, le travail artistique et littéraire a la capacité de modifier les complexions et de mobiliser les corps. Rendre véritablement "inaudible" (ou audible, c'est selon) une langue qui nous assujettit nécessite, en d'autres termes, une création de formes; en employant le lexique spinoziste qu'il affectionne, Lordon plaide pour des "machines affectantes" 19 ou des "prothèses passionnelles" ${ }^{20}$. En somme, le politique est inséparable d'une poétique.

10 Afin de mettre en pratique (de manière expérimentale) sa théorie des affects, Lordon publie en 2011 une Comédie sérieuse sur la crise financière en quatre actes et en alexandrins ${ }^{21}$. Si Nathalie Quintane, dans Tomates (2010), critiquait le fait qu'en France, la révolution se dît toujours dans un langage daté (celui du XVIII e siècle), occultant ce faisant son actualité et empêchant son retour ${ }^{22}$, Lordon dramatise cette logique au point de la renverser. Sa pièce met en relief les motifs de la crise de 2008 en exposant les coulisses du pouvoir : les banquiers, les "grands journalistes", le président de la République et ses conseillers manigancent à huis clos contre les citoyens - en vers de douze syllabes. 
La versification classique, imbibée des privilèges "naturels" de l'Ancien Régime, fait apparaître le pouvoir étatique, médiatique et financier comme une monarchie (ou une triarchie) absolue - le président se fait appeler altesse et traite le peuple de gueux ${ }^{23}-$, parfaitement indifférente aux conditions de vie du plus grand nombre. Dans Le grand retournement, l'adaptation filmique de la pièce, Gérard Mordillat accentue encore davantage l'écart entre la population et la sphère du pouvoir repliée sur elle-même en juxtaposant aux images lisses de la fiction des séquences documentaires figurant des tentes de sans-abris.

11 Le choc langagier qui résulte du recours à la forme classique met à distance le langage économiste imposant et imposé. Les expressions fétiches de ce discours ("moralisation", "rigueur", "loi du marché", "modernité") sont reprises sur un mode bouffon afin d'en exhiber la vacuité essentielle et nous libérer de leur emprise. Après avoir renfloué les banques pour leur éviter la faillite, l'État français se retrouve gravement endetté; les banquiers proposent alors au président la solution miracle: "Monsieur le président, prenez le bon chemin : / La rigueur aujourd'hui, la croissance demain" (CS: 109). En deux lignes, Lordon synthétise l'impératif qui commande l'économie depuis plusieurs décennies. Cette rigueur nécessaire imposée aux citoyens contraste pourtant radicalement avec la vie de luxe que continuent à mener les financiers - "Savez-vous qu'à Wall Street tout comme à la City / L'époque est revenue de toutes les folies ?" (CS: 84) Et lorsqu'un banquier parle de "rigueur durable" (CS: 113), l'un des conseillers du président - la seule voix de la raison dans la pièce - note l'“astucieux pillage de la langue écolo" (CS : 114) et déclame :

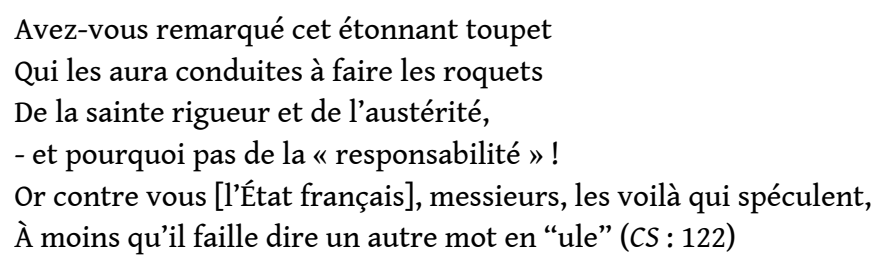

En suscitant le rire, le décalage entre un propos éminemment actuel et une forme désuète rompt l'enchantement des paroles du pouvoir, comme en témoigne ce passage où un banquier angoissé voit sa fin arriver : "Ma banqu', ma vie, mon œuvre, tout va donc y passer / Parachute doré, où est donc ta poignée ?” (CS : 25, nous soulignons). Le rire est jaune, car la transposition d'un trope financier dans des vers raciniens en fait apparaître le caractère à la fois grotesque et insupportable, d'autant que Lordon brise l'évidence de la métaphore en lui conférant des poignées. Plus tard, lorsque le conseiller éclairé annonce au président les risques d'un sauvetage des banques sans contrepartie et demande leur nationalisation, ce dernier est outré et éructe, comme un réflexe pavlovien, "pur communisme", "Corée du Nord" (CS: 74). Avant d'être renvoyé, le conseiller lance une dernière tirade qui présente le chef de l'État comme un enfant roi perdu dans un monde qu'il ne comprend pas :

Voilà c'est reparti, le président bébé

De nouveau a vagi, et ses mains remué.

Amenons les joujoux et surtout la totoche,

Il n'y a pas d'autre voie pour parler à un mioche. (CS : 69)

En suscitant des affects opposés qui se renforcent l'un l'autre - la jubilation et le dégoût, le rire et l'opprobre -, la pièce de Lordon est moins une entreprise de vulgarisation économique qu'une machine à décrédibiliser ceux qui considèrent que le monde leur est dû. En cela, cette comédie au style burlesque est tout à fait sérieuse : elle 
désamorce le langage de la finance, elle en rompt le charme. Comme une conséquence de cette neutralisation linguistique du pouvoir et de son monde, les dernières pages s'achèvent sur une France qui se soulève : "Vous êtes étonnés : le peuple est en pétard / La vraie surprise étant qu'il s'y mette si tard / [...] Vienne l'abus de trop ou l'incrément odieux / Et le seuil est franchi, et soudain tout prend feu" (CS : 126).

\section{Poétiser la sécurité} certain langage imposé en nouant, mais selon des modalités tout-à-fait diverses, l'expérimentation formelle et l'engagement politique. À l'instar de la pièce de Lordon, son récit - il faudrait sans doute trouver un terme plus adéquat pour le qualifier ${ }^{24}$ s'achève sur un soulèvement populaire, comme si, ici encore, la poésie (celle qui s'attache à rendre inopérantes les paroles du pouvoir) libérait des possibles et mettait en mouvement des forces jusque-là paralysées.

Un narrateur à peine déterminé, sans doute jeune, mais dont on ignore le sexe et le prénom, passe ses journées à fumer du cannabis, à manger des bananes, à flâner, à écouter Tupac - et à lire, entre autres, Victor Klemperer. Le nom du philologue apparaît à trois reprises dès la première page, juste après une remarque sur la situation actuelle : "Il n'y a pas beaucoup de poésie en ce moment, j'ai dit à mon père" (PE: 9). Tout au long du récit, le narrateur relit l'essai de Klemperer afin d'y trouver des clés pour comprendre le "durcissement" ( $P E: 10)$ de l'époque et l'“ambiance nouvelle" (PE: 9) qui l'accable. L'atmosphère étouffante de l'état d'urgence, les militaires qui patrouillent dans les rues, les violences policières et la "loi Travail" (nous sommes donc en 2015/2016) est rendue sensible par le discours du père, avec lequel le narrateur est en conflit.

Alors que ce dernier ne se résout pas à chercher un travail et vit "de façon éhontée" ( $P E: 59)$ sur les droits d'un mauvais roman écrit sous pseudonyme, le père est simultanément universitaire, chef d'orchestre, économiste, généticien, intellectuel, humanitaire (et il possède un $4 \mathrm{X} 4$ ). Moins un personnage qu'un symbole de pouvoir, il représente la position sociale établie, l'argument d'autorité qui disqualifie avec condescendance - "sais-tu au moins ce qu'est la poésie ?" (PE : 10). Contrairement aux paroles tâtonnantes et fragiles du narrateur, le discours du père, proféré "sur le ton habituel des cours magistraux" ( $P E: 12)$, impose, oblige et empêche. Cette figure paternelle est en même temps intériorisée ; elle a colonisé le cerveau du narrateur et le brutalise dès qu'il a des idées qui ne lui conviennent pas : "il foutait des coups de pied dans mes deux hémisphères, contre les tempes, [...] mon cerveau douloureux criait D'accord Papa" (PE : 23).

17 À travers le discours de ce personnage-surmoi, Lefebvre expose le caractère oppressant et parfois ridicule de ce que le narrateur nomme le "langage fabriqué" ( $P E: 71)$, c'est-àdire un ensemble d'expressions et de mots nouveaux qui se substituent à la langue maternelle. Il est significatif en ce sens que la mère du narrateur soit "morte il y a pas mal d'années" ( $P E: 55)$. Au début du texte, par exemple, le père affirme que la France doit s'engager dans "la course mondiale du monde mondialisé" (PE : 13). Tout en créant un effet comique, le pléonasme dépouille le mot de son éclat et en souligne la vacuité. Un peu plus tôt, le père s'en prenait au constat de manque de poésie en employant un langage quantitatif : "même si des experts en poésie pouvaient évaluer un taux de 
poésie et constater une tendance à la baisse, comment peux-tu établir que cette baisse tendancielle du taux de poésie a un quelconque rapport avec ce qui se passe en ce moment?" (PE: 10). Lefebvre dénonce ainsi l'omniprésence de la terminologie économiste, qui s'applique indistinctement à tous les domaines de la vie: chaque énoncé doit être intégré à la rationalité économique sous peine d'être invalidé. Le télescopage de ce discours avec le monde de l'inquantifiable (l'impression, la poésie) suffit à en faire apparaitre le caractère incongru. À chaque fois, les paroles affirmatives du père se neutralisent elles-mêmes; le narrateur n'argumente jamais et se contente d'un acquiescement minimal, presque bartlebien.

Un peu plus loin, la voix paternelle déclare que la poésie doit œuvrer "dans le cadre sacré de la défense nationale de l'Europe libérale" et est "priée de défendre librement la liberté de l'économie de marché" (PE: 13). Le discours sécuritaire s'allie ici au discours néolibéral pour soumettre toute action à ses exigences - la littérature doit, comme toute institution, se mettre au service de la "Liberté". La répétition de ce mot comme un mantra, avec un L majuscule et dans toutes ses variantes (libéral, librement), lui ôte à la fois sa pertinence et sa validité. À une longue tirade au cours de laquelle le père martèle des mots comme "Civilisation", "République" et "Démocratie", le narrateur oppose un simple "Ça veut dire quoi, Papa?" (PE:13), qui exhibe ce discours comme un tissu de clichés vides de sens - mais dotés d'une efficacité, car ils permettent à celui qui les emploie de se rendre inattaquable en se faisant passer pour progressiste. Quelques chapitres plus tard, Lefebvre montre que ces paroles de vertu majuscules s'effondrent dès que des citoyens manifestent leur désaccord avec le gouvernement : "il y avait l'eau des canons sous la pluie et une compagnie républicaine en pleine action de sécurité qui est, d'après le ministère de l'Intérieur, la première des libertés car tu dois travailler" (PE : 93).

19 Précisons toutefois que la parole du père, aussi autoritaire soit-elle, n'est pas assimilable à une idéologie d'extrême droite. Quand sur un marché, une jeune fille à l'air affable propose au narrateur un tract ("on est chez nous"), le père la traite aussitôt de fasciste. Lefebvre indique ainsi, d'une part, que le langage "fabriqué" contemporain est plus difficile à combattre et à démasquer que la LTI, car il s'avance sous couvert de démocratie et de liberté, et d'autre part que ceux qui l'emploient ne se rendent pas compte de ses rapports avec un certain discours fascisant ${ }^{25}$. Après avoir longuement étudié Klemperer, le narrateur en conclut ainsi, dans l'un des passages les plus didactiques du livre :

Ce qui me semblait résonner avec l'époque nazie où l'empoisonnement de la langue était une activité de propagande politique à plein temps, c'était la gravité des formules toutes faites de la France unie contre le terrorisme, qui encourageait la propagande sur les réseaux sociaux, dans la presse locale et nationale, [...] des vociférations de préférence nationale et de République laïque historiquement chrétienne, et l'amplification [...] des formules de haine contre tout ce qui n'est pas soluble dans le maintien de l'ordre et du pouvoir d'achat des classes moyennes. (PE : 72)

20 Au fur et à mesure de ses lectures, de ses observations et de ses réflexions, le narrateur se détache progressivement du langage de son père et de tous les "modes d'expression [qui] inoculent chaque jour leur poison dans [s]a façon de penser" (PE : 70).

21 Le personnage découvre plusieurs antidotes à ce "poison" ${ }^{26}$, à commencer par la drogue. Dès qu'il fume, son père quitte la scène. Désinhibée, la pensée s'émancipe de sa tutelle discursive; des éclats de poésie et de vérité sont alors libérés : "La catégorie 
socio-professionnelle n'a pas de réalité, [...] le vocabulaire s'aveugle, le sujet est surveillé, le verbe ne fait rien, le complément est à ceux qui peuvent se le payer" (PE : 15). Mais rapidement, l'effet du cannabis s'estompe et la parole écrasante du père resurgit, tout aussi imposante qu'avant. Le second antidote, plus simple, consiste à adopter un rapport naiff à la langue étouffante : "je me demandais ce que voulait dire chez nous. Peut-être que chez nous était ce carrefour où nous étions, entre la pharmacie, le Crédit Agricole, l'épicerie arabe et la boutique de fringues, ou toute la rue piétonne" $(P E: 20)$. Interroger les expressions figées sur un mode enfantin en fait entendre la bêtise et met au jour les sous-entendus tapis derrière leur apparente banalité.

Petit à petit, le narrateur se rend compte que l'antidote le plus puissant contre le langage du père est la poésie. Comme s'il voulait en pallier l'absence constatée au début du récit, il décide de devenir lui-même poète - d'autant qu'il s'agit d'un "emploi dans rien sous le contrôle de personne" (PE : 35$)$. Il faut préciser toutefois que "poésie" prend ici un sens bien particulier. Le narrateur dénonce la poésie désintéressée et contemplative avec "ses vers alignés comme des petits cercueils au printemps des poètes, [qui] ressemble à un morceau de secteur culturel avec les barrières et le niveau de sécurité, fuck that fake" (PE : 59). Il s'agit de chercher la poésie dans des lieux où elle n'est pas attendue, où elle n'est pas une "langue cultivée" $(P E: 87)$ parmi d'autres. Le narrateur la rencontre notamment dans une prose pleine de fautes d'orthographe sur le forum d'un site ("feminin.com"), où une jeune femme encourage une autre à porter plainte pour viol en racontant sa propre expérience. Alors, "c'est comme si ça se mettait à bouger quelque chose, comme si d'un coup la vulnérabilité devenait la raison même d'une souveraine beauté" ( $P E: 60$ ). En somme, est poétique toute parole non préformatée qui échappe aux dispositifs visant à l'encadrer ${ }^{27}$ et qui fait bouger quelque chose.

Pour transmettre (et comprendre lui-même) sa conception de la poésie, le narrateur propose une série de "leçons", dont la plus importante est peut-être la huitième : "Poètes, ne laissez pas empoisonner les mots et tenez-vous loin de la littérature" (PE : 73). La tâche principale du poète consiste à veiller sur le langage commun afin d'empêcher qu'il fasse l'objet d'une "fabrication" néfaste; une telle poésie se situe nécessairement en dehors de la "littérature", si par ce terme on entend une sphère culturelle séparée, autonome et institutionnalisée. L'une des armes poétiques ${ }^{28}$ employées par le narrateur est la versification de textes réels. Par exemple, il découpe en vers un passage de la "loi Travail" de 2016 : "Le salarié est / À la disposition de l'employeur et / Se conforme à ses directives / Sans pouvoir / Vaquer / Librement / À ses occupations / Personnelles" (PE: 45). Les mots ainsi détachés les uns des autres modifient le rythme de lecture; accentués et séparés du flux discursif, ils font apparaître la violence législative dans toute sa nudité. Cette décontextualisation neutralise la légitimité pompeuse de la loi et crée une distance qui rend possible sa suspension. Ailleurs, un appel au recrutement de l'armée - il s'agit ici encore d'un collage, sans la référence au texte réel ${ }^{29}$ - est mis en pièces de la même manière : "Des jeunes motivés qui sont / À la fois / Endurants / Courageux / Assez autonomes / Et qui font preuve de rusticité" ( $P E: 36)$. Après cette mise en vers, le narrateur poursuit le travail de déconstruction en reprenant le dernier adjectif pour le substantiver : "Les rustiques de l'armée de terre sont jeunes et motivés" (PE: 37). Ainsi réagencé (sémantiquement, rien n'est modifié), l'appel apparaît à la fois risible et inquiétant: l'une des qualités demandées aux militaires qui patrouillent sur les boulevards est le 
manque de finesse. Le travail poétique permet ainsi de mieux saisir la dangerosité d'un certain langage, tout en désactivant son pouvoir.

Malgré l'ambiance étouffante de l'époque, l'humeur du narrateur s'améliore au fil du texte: "En mars, j'allais bien" ( $P E: 90)$. Non seulement il se libère de l'emprise paternelle grâce à l'écriture, mais l'arrivée du printemps coïncide avec "les mouvements sociaux et les retours d'oiseaux" (PE: 90). Grâce au surgissement de nouvelles luttes sociales (rappelons que Nuit debout voit le jour en mars 2016 à la faveur de la contestation de la loi Travail), le narrateur sort de son repli solitaire. Et quand, à la fin, réjoui, il revoit son constat initial - "il y a pas mal de poésie en ce moment" ( $P E: 102)$ - pour la première fois le père ne répond pas. "Tu peux toujours bavarder sur le langage, qu'est-ce que ça va changer ?” (PE : 70), demandait-il un peu plus tôt. Tout, semble lui rétorquer la fin du récit.

\section{Exhiber la violence}

Frédéric Lordon et Noémi Lefebvre engagent la littérature en inventant des formes dont l'énergie propre vise, en un même geste, à mettre à nu un langage jugé nocif et à en rompre l'enchantement. Loin de la gravité des romans à thèse, leurs œuvres à la fois joyeuses et optimistes trouvent la radicalité dans la forme, et insistent sur le caractère fondamental, du point de vue de la lutte émancipatrice, du rapport poétique au langage. Tel qu'ils le conçoivent, le poète a un rôle politique majeur : en faisant éclater un consensus linguistique oppressant, il ouvre la langue, la communauté et le monde (indissociablement liés) à de nouveaux agencements possibles. Le travail de Nathalie Quintane s'inscrit dans cette même logique. Dans Ultra-Proust, elle note que "le 'travail de la langue' est une chose absurde quand on ne comprend pas qu'il ne se réduit pas à lui-même comme objet d'autocontemplation" ${ }^{30}$. Sa poétique s'attaque au langage dévoyé avec des procédés similaires à ceux employés par Lordon et Lefebvre : posture naïve, collage, ironie, incongruité, et ainsi de suite ${ }^{31}$ - autant de façons de défamiliariser le lecteur en faisant trembler un certain état de la langue.

Les enfants vont bien, paru en 2019, se fixe le même but - exposer/désactiver le langage "de ceux qui entendent nous gouverner par la parole" ${ }_{32}-$, mais simplifie radicalement les moyens d'y parvenir. Il s'agit en effet d'un texte qui repose sur un travail poétique minimal : l'écrivain se contente (mais cela suppose une acuité remarquable) d'extraire des discours contemporains un ensemble de propositions, tantôt une phrase, tantôt quelques mots, qui sont ensuite disposés sur la page sans référence ni commentaire. En d'autres termes, les collages ne sont plus intégrés à un tissu narratif, mais sont exhibés isolément (un seul par page), pour éviter sans doute que le discours de l'auteur ne se substitue au flux discursif, dont ils ont été extraits précisément pour mieux les voir. Dans sa préface, Nathalie Quintane explique ainsi que le "parti pris narratif" (EB:7) de ses livres précédents lui semblait insuffisant à "rendre compte de la violence" (EB:7) que subissent notamment les réfugiés en France. Par l'accumulation en mosaïque de ces paroles fragmentaires-Quintane se situe elle-même dans une lignée d'auteurs pratiquant le "montage" $33(E B: 7)$-, son dernier ouvrage se présente comme un diagnostic d'une certaine France des "années 10".

Toutes les paroles citées dans le livre ne se valent toutefois pas. L'origine des fragments est signalée spatialement et typographiquement. Les mots situés en haut de la page, dans une police imposante, ont été proférés par des hommes politiques. Quintane 
indique ainsi la hiérarchisation des discours : ceux-ci parlent tellement fort qu'on ne peut pas ne pas les entendre. L'absence de référence, tout en empêchant l'argument d'autorité, souligne le fait que ces phrases ne sont pas idiosyncrasiques: par leur diffusion à grande échelle, elles risquent d'intégrer la langue commune - il est donc d'autant plus urgent de les mettre hors-jeu. En isolant ces éclats de violence de leur contexte, Quintane nous montre à quel point ils sont "toxiques". Certaines propositions semblent tirées tout droit de la novlangue orwellienne - "Il ne peut y avoir d'humanité s'il n'y a pas de fermeté" (EB :

38) -, d'autres soulignent la violence à peine voilée du discours quantitatif - "Il y a les sans-papiers et les Roms. J'ai mon quota," (EB: 152) -, d'autres encore reprennent une simple expression qui, sous couvert d'une métaphore classique, écrase toute forme de générosité et d'altruisme : "les belles âmes qui" (EB : 191).

Un peu plus bas sur la page, en italique, Quintane place des extraits de textes législatifs qui résonnent avec les paroles politiciennes: par cette simple juxtaposition, l'apparente neutralité de la loi est mise à mal. En milieu de page, on trouve ensuite le discours bureaucratique des centres d'accueil, qui se situe à mi-chemin entre une rationalité froide et quantitative et une réelle volonté de soutien, comme en témoigne ce fragment ambigu: "augmenter notre efficacité et donner les meilleures chances à nos amis" (EB: 66). Plus bas encore, en gras, des extraits de presse décrivent l'impensable sur le mode du constat, dans un langage tantôt plat, tantôt sensationnaliste : "migrant percuté : une personne en garde à vue" (EB: 161); "un homme brûlait" ( $E B: 198)$; "Il avait 28 ans, il était gambien" ( $E B: 236)$. Enfin, dans une police minuscule, comme une note de bas de page à ces mots oppressants, Quintane fait entendre les paroles fragiles, presque chuchotées, de ceux qui aident les réfugiés en marge de tout organisme étatique - "parfois on ose un geste, un café" (EB : 113). La rencontre d'une telle voix avec la grandiloquence du pouvoir, la sécheresse de la loi, la froideur bureaucratique et les constats de la presse suffit à mettre en lumière la charge de violence contenue dans l'ensemble de ces discours - "à nous de parler et d'écrire autrement" (EB: 11).

Contrairement à une tradition poétique qui se détourne du langage commun parce qu'il serait mort, appauvri ou inexpressif, les trois écrivains envisagés prennent sa défense et luttent contre les paroles "fabriquées" qui le colonisent par répétition médiatique. C'est précisément en raison de leur puissance - la communauté à laquelle Quintane croyait appartenir en a fait les frais - qu'il faut travailler les à faire dérailler, à les rendre inopérantes. En simplifiant, on pourrait distinguer trois opérations critiques par rapport à un langage imposé $: 1^{\circ}$ rétablir une convention perdue $; 2^{\circ}$ inventer de nouvelles conventions, de nouveaux usages $; 3^{\circ}$ suspendre la convention actuelle afin d'ouvrir des possibles indéterminés. En somme: restituer, constituer, destituer. Les trois textes envisagés s'inscrivent principalement dans cette dernière veine - le livre de Quintane, qui met à nu un discours répressif en se passant de tout commentaire, en est l'expérience paroxystique. Évitant toute posture de surplomb (l'écrivain se situe ici du côté du plus grand nombre), ces auteurs évitent de "conquérir" la langue et leurs attaques sont obliques: "Poètes, ne soumettez personne à la raison d'un père" (PE: 29) est la leçon numéro 1 du narrateur de Lefebvre. En somme, à la violence d'une poétique constituante (celle de la LQR, par exemple), ces écrivains opposent humblement ce qu'on pourrait appeler une poétique destituante ${ }^{34}$. 
doit à Giorgio Agamben d'avoir théorisé le concept de destitution, nécessaire selon lui pour repenser la révolution elle-même. À l'aune de l'idée benjaminienne de "violence pure", il s'agit pour lui d'envisager une dissolution d'un pouvoir souverain sans réinstitution ultérieure : "une puissance purement destituante, c'est-à-dire qui ne se résolve jamais dans un pouvoir constitué" ${ }^{35}$. Éviter de prendre le pouvoir, refuser de constituer, car ces gestes réintroduisent nécessairement une forme de domination; la destitution ouvrirait au contraire à une politique différente, qui échapperait à la violence et suspendrait le droit. Agamben ne parle jamais de "poétique destituante", mais il avance brièvement, dans Création et anarchie, l'idée d'une "poétique du désœuvrement" ${ }^{36}$. Lorsqu'il donne des exemples concrets pour expliquer cette notion d'inoperosità, de désœuvrement, le philosophe évoque toujours d'abord la poésie. Le poème est l'acte qui, par le langage, désœuvre le langage. C'est l'acte qui "rend inopérante la langue" ${ }^{37}$ (dans son aspect notamment communicationnel) et l'ouvre à d'autres usages - le poème rend possible, par exemple, la contemplation de la langue en tant que telle, et cette contemplation suspend toute assignation de l'humain à une œuvre particulière ${ }^{38}$. Dès lors, tout poème digne de ce nom (Agamben cite Dante, Leopardi, Hölderlin, Bachmann, Rimbaud) est à chaque fois le désœuvrement d'une langue particulière, et en ce sens, l'expression "poétique du désoeuvrement" est tautologique. C'est là que se situe la différence avec la poétique destituante qui définit selon nous les trois œuvres envisagées.

Pour en saisir le sens, il nous faut faire un dernier détour, par une notion que développe Agamben dans Le règne et la gloire. Dans cette archéologie du gouvernement, le philosophe découvre que ce qui fonde la souveraineté, ce qui lui confère son pouvoir, c'est d'abord et avant tout la glorification. Sans la prière et la liturgie, Dieu serait déchu de son trône ; sans l'acclamation publique, le fascisme serait déchu de son pouvoir. La prière, le chant et l'acclamation ${ }^{39}$ sont autant de paroles qui, tout en tournant à vide, ont une efficacité propre : elles garantissent secrètement la légitimité du souverain. Or pour Agamben, la sphère de la gloire "ne disparaît pas dans les démocraties modernes, mais se déplace simplement dans un autre contexte, celui de l'opinion publique. Si tel est bien le cas, le problème aujourd'hui si discuté de la fonction politique des médias dans les sociétés contemporaines acquiert une nouvelle signification et une nouvelle urgence" ${ }^{\prime 4}$. Si le philosophe a raison, si l'ancienne fonction de glorification n'a pas disparu et survit aujourd'hui grâce aux médias ${ }^{41}$, alors la littérature a bien une puissance destituante: désoeuvrer le langage "médiarchique" ${ }^{42}$ revient à rendre la gloire inopérante et, par conséquent, à ôter au pouvoir sa légitimité en l'exposant comme essentiellement an-archique, sans fondement.

Une poétique destituante est une poétique qui se donne pour horizon le désœuvrement d'un langage fabriqué, dans le but de neutraliser le pouvoir souverain qu'il institue. Il nous semble que c'est sur cette voie qu'aujourd'hui plusieurs écrivains s'engagent. 


\section{NOTES DE FIN}

1. Ce dernier est nommé président de la communauté juive de Dresde par les Nazis.

2. Victor Klemperer, LTI, La langue du III ${ }^{e}$ Reich, Paris, Pocket, 2003, p. 249. Le livre est publié en français pour la première fois en 1995.

3. Ibid., p. 250

4. Klemperer lui-même n'y échappe pas toujours. Fuyant Dresde en 1945, il a peur d'être arrêté et dit craindre que la Gestapo ne "vienne [1]e chercher" [mich holen], et se reprend immédiatement : “ 'Venir me chercher !' Voilà que je mets moi aussi à parler dans cette langue !”, ibid., p. 346.

5. Nathalie Quintane, Les années 10, Paris, La fabrique, 2014, p. 180.

6. Éric Hazan, LQR. La propagande au quotidien, Paris, Raisons d'agir, 2006, p. 12.

7. Ibid., p. 14.

8. Ibid., p. 21.

9. Ibid., p. 16.

10. François Cusset a récemment mis en évidence le caractère performatif de cette langue : "Une guerre s'appelle 'frappe chirurgicale' ou 'ingérence humanitaire', un chômeur est une personne 'en recherche d'emploi', l'éviction de milliers de salariés est un 'plan de restructuration', la lutte acharnée pour l'emporter sur ses rivaux 'la réalisation de soi", dans La droitisation du Monde, Paris, Textuel, 2016, p. 103. On peut également lire le diagnostic du Comité invisible: "Il y a la pratique réelle d'un côté, et de l'autre le discours, qui en est le contrepoint implacable, qui est la perversion de tous les concepts, la tromperie universelle de soi-même et des autres", dans Maintenant, Paris, La fabrique, 2017, p. 10. Voir enfin Frédéric Joly, La langue confisquée : lire Victor Klemperer aujourd'hui, Paris, Premier Parallèle, 2019.

11. Victor Klemperer, op. cit., p. 40.

12. Jacques Derrida, Le monologuisme de l'autre ou la prothèse d'origine, Paris, Galilée, 1996, p. 15.

13. On ne peut évidemment s'empêcher de penser ici à la "novlangue" inventée par George Orwell en 1948.

14. Victor Klemperer, op. cit., p. 41.

15. Éric Hazan, op. cit., p. 121.

16. Nathalie Quintane, Les années 10, op. cit., p. 181.

17. Frédéric Lordon, "Surréalisation de la crise", post-scriptum à D'un retournement l'autre. Comédie sérieuse sur la crise financière en quatre actes et en alexandrins, Paris, Seuil, 2011, p. 131. Dorénavant $C S$.

18. Du moins dans sa version scientiste.

19. Frédéric Lordon, Les affects de la politique, Paris, Seuil, 2016, p. 61.

20. Ibid., p. 170.

21. Précisons que l'attention au discours, à la "construction rhétorique", traverse toute l'œuvre de Lordon, et est en tant que telle inséparable de sa vocation critique. Cette pièce n'est donc pas une tentative isolée dans son travail. Voir François Provenzano, Antoine Janvier, "Critique du capitalisme néolibéral et travail rhétorique chez Frédéric Lordon: un discours d'affectation", Dissensus, $\mathrm{n}^{\circ} 6$, juillet 2016, p. 42-54.

22. "[En] France, on sait bien comment sonne la Révolution, parce qu'on connaît sa langue : c'est celle du XVIII ${ }^{e}$ siècle. Comment une prose classique française fait-elle entendre le son du canon en 2009 ? Périodes et lexique typique (filles au lieu de putes), pensée construite, rythme tenu et bien frappé sur sa fin, latin. Nous lirions en perruque", Nathalie Quintane, Tomates, Paris, Points, 2014, p. 39-40.

23. Il est clair que le président idiot de Lordon est une caricature de Nicolas Sarkozy, en poste au moment de la publication: “Comprendras-tu, crétin, qu'avecque celui-là / Qui est un peu petit, tu 
ne parleras pas / Sans mettre ce qu'il faut de longues platitudes / Qui disent la grandeur, la taille et l'altitude" (CS: 43 , nous soulignons).

24. Le narrateur déclare ainsi: "je ne sais pas écrire une histoire en entier, je veux dire à l'ancienne, avec sa fin qui a fait le tour et tout ce qui s'ensuit", Noémi Lefebvre, Poétique de l'emploi, Paris, Gallimard, 2018, <Verticales>, p. 58. Dorénavant PE.

25. "Pour autant ce n'était pas le III ${ }^{\mathrm{e}}$ Reich, ça je tiens à le dire. Il faut tout de même pas chier" (PE : 73).

26. Klemperer parle souvent de la LTI en ces termes : “Combien de concepts et de sentiments n'at-elle pas souillés et empoisonnés!" Victor Klemperer, op. cit., p. 24.

27. L'école et l'université sont critiquées dans le récit, parce qu'ils enferment les grands textes et en désactivent la puissance en les patrimonialisant - à l'université, le narrateur a eu un professeur "qui ouvrait des perspectives étranges, en fermait beaucoup" (PE : 34 ). Voir également à ce propos l'essai de Nathalie Quintane, Ultra-Proust, Paris, La fabrique, 2018.

28. Cet imaginaire de la poésie comme arme a fait l'objet d'un recueil d'essais publiés aux éditions La fabrique : Jean-Christophe Bailly, Jean-Marie Gleize (et alii), "Toi aussi tu as des armes". Poésie et politique, Paris, La fabrique, 2011.

29. Il s'agit pourtant bien d'un véritable appel: https://www.aumilitaire.com/communaute/ topic/16005-a-lire-recrutement-13\%C3\%A8me-rdp/page/5/ (page consultée le 20/11/2019).

30. Nathalie Quintane, Ultra-Proust, op. cit., p. 54.

31. Voir Justine Huppe, "L'insurrection qui vient par la forme. Politique des styles chez Nathalie Quintane", COnTEXTES, n²2, 2019, url : <https://journals.openedition.org/contextes/6975> (page consultée le 15/04/2020).

32. Nathalie Quintane, Les enfants vont bien, Paris, POL, 2019, p. 7. Dorénavant EB.

33. Dans le prologue, Quintane rend hommage à Charles Reznikoff, à Heimrad Bäker et et Jacques-Henri Michot.

34. Cette méfiance envers l'affirmation est thématisée dans Poétique de l'emploi : "J'ai dit ça comme une impression ou peut-être un avis et pas comme une idée, enfin rien qui s'impose" ( $P E$ : 9).

35. Giorgio Agamben, L'usage des corps, dans Homo Sacer. L'intégrale 1997-2015, Paris, Seuil, 2016, p. 1324.

36. Giorgio Agamben, Création et anarchie. L'œuvre à l'âge de la religion capitaliste, Paris, Rivages, 2019 , p. 50. Notons toutefois qu'Agamben entend le terme "poétique" d'abord dans un sens très large (le poiein grec, la création humaine), avant d'envisager, dans un second temps, la poésie en tant que telle.

37. Giorgio Agamben, Le règne et la gloire, op. cit., p. 641.

38. Le philosophe considère qu'il faut, contre la tradition occidentale d'origine aristotélicienne, envisager l'homme comme fondamentalement désoeuvré, sans œuvre. Or cette absence d'œuvre est sans cesse capturée, assignée par un pouvoir souverain à des œuvres qui la voilent. On voit bien ici le rapport avec le narrateur désoeuvré de Lefebvre.

39. "[La] langue qui tourne à vide comme forme suprême de glorification. L'hymne est la désactivation radicale du langage signifiant, la parole rendue absolument sans emploi, et pourtant conservée comme telle dans la forme de la liturgie", Giorgio Agamben, Le règne et la gloire, op. cit., p. 627.

40. Ibid., p. 645.

41. Les paroles "fabriquées" y acquièrent leur puissance par répétition incessante - Yves Citton parlerait de "frayages". Notons par ailleurs qu'il y a une différence majeure entre la glorification divine et la glorification médiatique : si dans le premier cas, les anges et les croyants louent le Dieu souverain, dans le second cas, le pouvoir souverain (les hommes politiques, notamment) se glorifie lui-même.

42. Yves Citton, Médiarchie, Paris, Seuil, 2017. 


\section{RÉSUMÉS}

À partir d'une lecture de trois textes récents, cet article s'attache à décrire une poétique particulière, qui met en rapport expérimentation formelle et engagement politique. D'un retournement l'autre (2011) de Frédéric Lordon, Poétique de l'emploi (2018) de Noémi Lefebvre et Les enfants vont bien (2019) de Nathalie Quintane inventent tous trois, selon des modalités différentes, des formes visant à rendre inopérante une langue (politique, médiatique, économique) jugée délétère. Nous montrons qu'à la suite des analyses de Victor Klemperer au sujet de la colonisation de la langue allemande par le national-socialisme, ces écrivains mettent en lumière les ruses et les tours de la "novlangue" contemporaine. Leurs textes ne se limitent toutefois pas à en constater les effets néfastes : tout en dévoilant le caractère pernicieux de cette langue qui se propage au point de devenir commune, ils visent, par le biais d'une série de formes poétiques que nous mettons en évidence, à lui ôter son pouvoir. Chez ces auteurs, la création littéraire est résolument branchée sur l'action politique. Dans la lignée des réflexions de Giorgio Agamben sur la révolution, nous proposons de qualifier leur poétique de destituante.

\section{INDEX}

Mots-clés : politique, destitution, radicalité, fascisme

\section{AUTEURS}

JULIEN JEUSETTE

Università degli Studi di Milano 